Dieter Bimberg, Gunter Larisch

\title{
Nanophotonics for a green internet
}

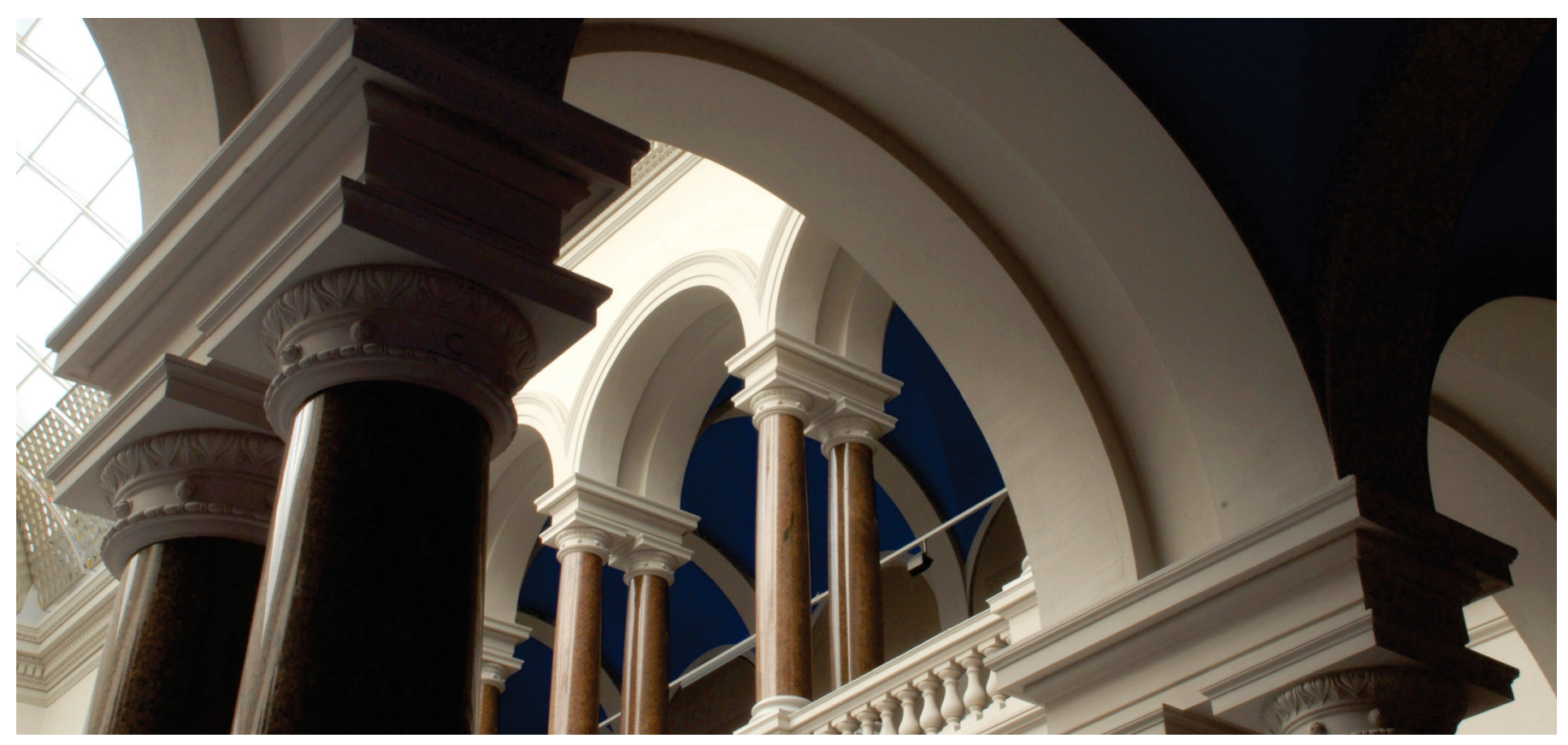

Bimberg, D. H., \& Larisch, G. (2019). Nanophotonics for a green internet. Proc. SPIE 10922, Smart Photonic and Optoelectronic Integrated Circuits XXI, 109220B (9 April 2019). https://doi.org/10.1117/12.2506015 


\title{
Proc. SPIE Conference 10922: Smart Photonic and Optoelectronic Integrated Circuits XXI 2019 Nanophotonics for a Green Internet
}

\author{
Dieter Bimberg ${ }^{\mathrm{a}, \mathrm{b}}$, and Gunter Larisch ${ }^{\text {a }}$, \\ "ar'Bimberg Chinese-German Center for Green Photonics" of the Chinese Academy of Sciences at \\ Changchun Institute of Optics, Fine Mechanics, and Physics (CIOMP), 3888, Dongnanhu Road, \\ Changchun 13033 People's Republic of China; ${ }^{b}$ Center of Nanophotonics, Institute of Solid State \\ Physics, Technische Universität Berlin, Berlin D-10632, Federal Republic of Germany;
}

\begin{abstract}
Energy efficient 200+ Gbit/s single fiber data transmission systems can be realized by wavelength multiplexing the directly modulated Vertical-Cavity Surface-Emitting Lasers (VCSELs) presented here, emitting at the four wavelengths $850 \mathrm{~nm}$, $880 \mathrm{~nm}, 910 \mathrm{~nm}$, and $940 \mathrm{~nm}$. Large energy efficiency defined by a heat to data ratio (HBR) of only $240 \mathrm{fJ} / \mathrm{bit} @ 50 \mathrm{~Gb} / \mathrm{s}$ is observed. Tuning the cavity photon lifetime is demonstrated to lead to an increase of the data rate in concert with a reduction of the HBR. The large linearity of our L-I-characteristics will allow easily higher order modulation rates. Our results might impact ongoing discussions of new physical layer standards for IEEE $802.3 \mathrm{~cm}$ and $802.3 \mathrm{~cd}$ coarse wavelength multiplexing standards across OM5 multimode fiber enabling up to $400 \mathrm{Gbit} / \mathrm{s}$ error-free transmission.
\end{abstract}

Keywords: Energy efficiency, optical interconnects, wavelength division multiplexing, vertical cavity surface emitting laser, single mode emission, physical layer standards.

\section{INTRODUCTION}

The energy required to transmit information as encoded optical and electrical data bits within and between electronic and photonic integrated circuits, within and between computer servers, within and between data centers, and ultimately across the globe from one point to another one clearly must be minimized. This energy spans from typically tens of picojoulesper-bit in data centers to well over tens of millijoules-per-bit for intercontinental distances. We seek to meet the exploding demand for information within the terrestrial resources available, try to reduce costs and thus become stewards of a perpetual Green Internet. The concept of a Green Internet implies a collection of highly energy-efficient, independent, and ubiquitous information systems operating with minimal impact on the environment via natural or sustainable energy sources. A key enabling optical component for the Green Internet is the vertical-cavity surface-emitting laser (VCSEL) employed in data centers. We present our latest results of research on energy-efficient VCSELs for wavelength mutliplexing, being sources for optical interconnects, operating at bit rates 50+ Gbps with energy efficiencies not far from the magic number of $100 \mathrm{fJ} / \mathrm{bit}$.

\section{STRUCTURE AND DESIGN OF THE LASERS}

Our VCSELs are grown by MOVPE on 0-degree off (001)-oriented GaAs substrates and emit at $850 \mathrm{~nm}, 880 \mathrm{~nm}, 910 \mathrm{~nm}$, and $940 \mathrm{~nm}$ at room temperature (RT). We use AlGaAs distributed Bragg reflector (DBR) mirrors with compositionallygraded interfaces. The quantum well active region consists of five compressively-strained InGaAs quantum wells surrounded by either strain-compensating GaAsP barrier layers or by unstrained AlGaAs barrier layers (depending on the desired emission wavelength), all in half-lambda-thick optical cavities. Details of the laser structure will be reported elsewhere.[1]

The VCSELs are processed in a double mesa design to ensure a good heat conduction out of the active region. GSG (Ground-Signal-Ground) contact pads enable the evaluation of a large number of devices of 256 design variations in a short time. The use of BCB (benzocyclobutene) reduces the parasitics by reducing the pad-capacitances [2]. A patented

\footnotetext{
*larisch@ciomp.ac.cn; phone: +49 1775667700
} 
optimization step, based on reflectance tuning of the out-coupling mirror by deposition of a dielectric, was used to increase the bitrate while reducing the energy consumption [3]. For optical and electrical confinement we employ oxide apertures fabricated by selective wet oxidation of $\mathrm{Al}_{0.98} \mathrm{Ga}_{0.02} \mathrm{As}$ layers [4]. An optical in situ-controlled in-house designed and built steam oxidation furnace was used to form the apertures with a tolerance of $0.5 \mu \mathrm{m}$. Due to a large variation of upper mesa diameters provided by our mask set, 16 different aperture diameters are available with every process run [3].

\section{STATIC PROPERTIES}

\subsection{Demands for energy efficient active cables}

The requirements for optical links will in the future not be satisfied only by achieving large bit rates in concert with low energy consumption. Integration of energy efficient driver circuits, VCSELs, optical waveguide, photodiodes and amplifiers is needed [5,6] (see Fig. 1). The electrical and optical properties of the laser must be matched to the other components.

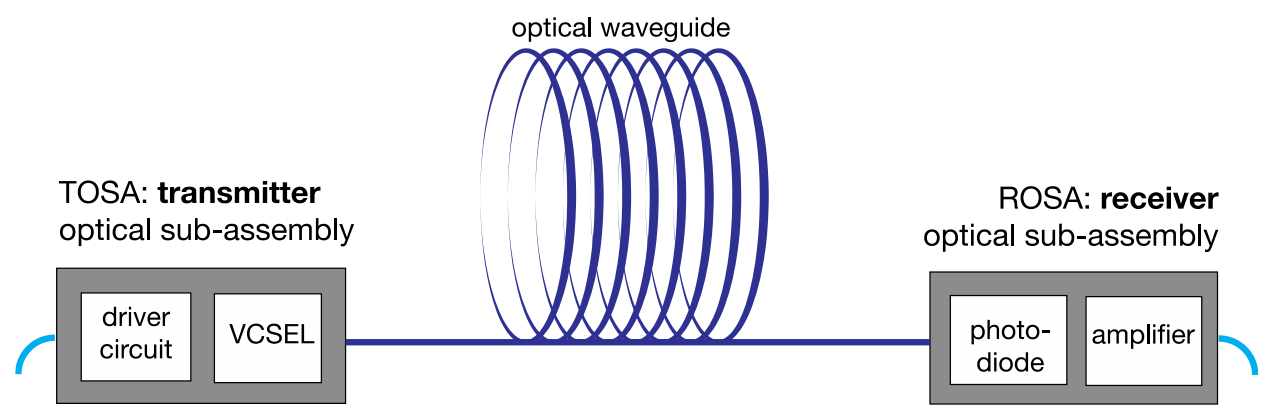

Fig. 1: Sketch of an optical link containing a driver circuit, a VCSEL, an optical waveguide, a photodiode, and an amplifier. Driver circuit and laser form the transmitter optical sub-assembly TOSA, amplifier and photodiode form the receiver optical sub-assembly ROSA.

\subsection{Large optical power output at low bias current}

A reduction of the VCSELs aperture size to reduce the input power consumption is only reasonable if the optical output power does not decrease too much. Our approach here is based on increasing the slope efficiency by employing our patented photon lifetime optimization step, leading to an increase of optical power for relatively small aperture diameter VCSELs at low currents [3]. A variation of 16 different aperture diameter VCSELs ranging between $\sim 1 \mu \mathrm{m}$ to $\sim 14 \mu \mathrm{m}$ provided by a top mesa size variation of our mask set allows to select lasers that satisfy the optical power demand of the photodiode at the lowest possible bias $I$ and threshold current $I_{\mathrm{th}}$. All lasers at all wavelengths have a very large slope efficiency of $1 \mathrm{~W} / \mathrm{A}$ with a very linear LI-curve [1]. So even small oxide aperture diameter VCSELs with single mode emission as shown in Fig. 2 have a large optical output power of nearly $3 \mathrm{~mW}$.

\subsection{Large extinction ratio due to large slope efficiency}

Downsizing our lasers reduces the bias current and thus allows downsizing the energy demand of the driver electronics too. Since the power consumption of drivers is presently a magnitude larger than the power consumption of the lasers [7] the total power consumption of the optical link will be reduced dramatically.A decrease of the aperture diameter results in a larger differential resistance. Since the driver electronics creates a certain voltage hub (e.g. $1 \mathrm{~V}_{\mathrm{pp}}$ ), a large slope efficiency results in a large optical modulation range and extinction ratio [8].

\subsection{Emission spectra}

The distance a signal can be transmitted across a fiber depends on the attenuation and the dispersion. The attenuation depends on the wavelength, and increases with the fiber length, Longer fibers need increased optical power. Dispersion 
increases spectral width at the receiver end and might lead to crosstalk. For narrow signal emission spectra crosstalk is eliminated. Our single mode emission lasers show large optical power compared to other single mode VCSEL approaches [9] and are well suited for high-speed data transmission across multimode fibers for typical data center distances of a few hundred meters.

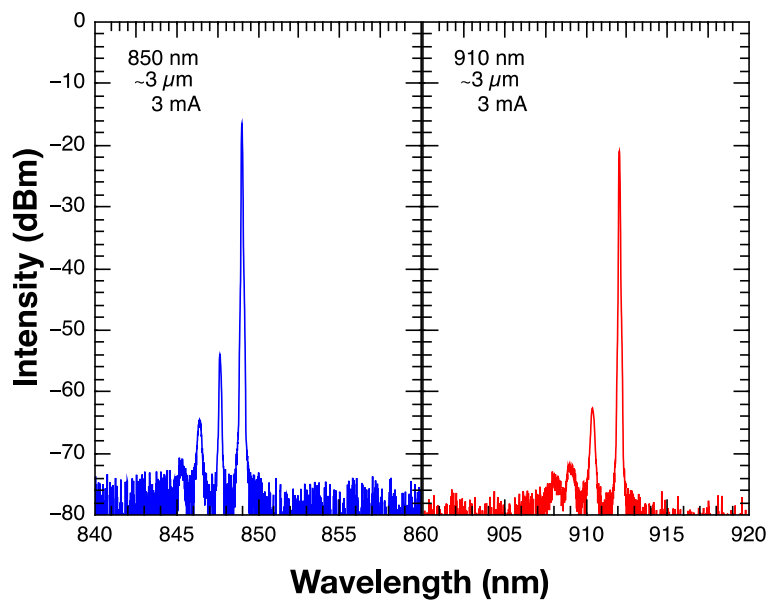

Fig. 2: Emission spectra of single mode emitting laser with optical power of $\sim 3 \mathrm{~mW}$ at the wavelengths $850 \mathrm{~nm}, 880 \mathrm{~nm}$, $910 \mathrm{~nm}$, and $940 \mathrm{~nm}$.

To increase data rates across single multimode fibers wavelength division multiplexing according to the new IEEE802.3cd and $\mathrm{cm}$ standards is discussed. Lasers with emission wavelengths of $850 \mathrm{~nm}, 880 \mathrm{~nm}, 910 \mathrm{~nm}$, and $940 \mathrm{~nm}$ might be used in such CWDM systems and were fabricated by us. For all four wavelengths almost identical L-I-V properties are observed. The typical emission spectra shown in Fig. 2 are also similar to each other. At all wavelengths, single mode emission with large optical output power was achieved for aperture diameters of $3 \mu \mathrm{m}$ and below. The thermal wavelength shift is $0.069 \mathrm{~nm} / \mathrm{K}$.

\section{DYNAMIC PROPERTIES}

\subsection{Laser physics and laser performances}

The most important application of the lasers presented here is large bit rate data transmission across optical fibers. The parameters defining their performance are the maximum error-free bit rate $B R$,

$$
B R=\text { number of transmitted bits/time }
$$

the energy to data ratio $E D R$

$$
E D R=V \cdot A / B R,
$$

with $V$ bias voltage and $A$ bias current as well as the more frequently used heat to bitrate ratio $H B R$

$$
E D R=V \cdot A / B R,
$$

with the optical power $P_{\text {opt }}[10]$.

We use standard single mode rate equations to describe the laser diode physics. Their solution yields the transfer function

$$
H(f)=\frac{f_{R}^{2}}{f_{R}^{2}-f^{2}+j\left(\frac{f}{2 \pi}\right) r} \cdot \frac{1}{1+j\left(\frac{f}{f_{p}}\right)}
$$

with the relaxation resonance frequency $\left(f_{R}\right)$, damping $(\gamma)$, and parasitic cut off frequency $\left(f_{p}\right)$. The value of $H(f)$ decreases with increasing frequency $(f)$ [11]. The frequency where $H(f)$ reduced by $3 \mathrm{~dB}$ as compared to the starting value defines the bandwidth $f_{3 d B}$. According to the Shannon-Hartley Theorem laser performance and laser physics are connected by the spectral efficiency $M[12]$, where 


$$
B R=M \cdot f_{3 d B} \cdot
$$

To increase the bitrate $B R$, the bandwidth $f_{3 d B}$ needs to be increased. Using highly strained multiple quantum wells in the active region increases the gain. Reducing the cavity length to $\lambda / 2$ and shrinking the aperture diameter reduces the active volume [13]. Both leads to an increase of the relaxation resonance frequency even at low bias currents. Furthermore, cavity photon lifetime tuning (e.g.by adding a thin layer of $\mathrm{SiN}$ to the top mirror surface) increases the $f_{3 d B} / f_{R}$-ratio and the bandwidth [3]. Assuming a reasonable spectral efficiency of $M=2 \mathrm{bit} / \mathrm{Hz}$ [13] bitrates larger than $50 \mathrm{Gbit} / \mathrm{s}$, are feasible. Exceeding $50 \mathrm{Gbit} / \mathrm{s}$ presently a drop of $M$ occurs in our experiments due to limitations of our equipment.

The maximum possible data rates which can be achieved depend equally on the properties of the passive fiber and the receiver. Receivers that are more sensitive than the presently commercially available ones enable larger distances and/or lower energy consumption per bit. Larger receiver cut-off frequencies enable additionally larger data rates. Despite these limitations, we observed error-free data transmission rates of $52 \mathrm{Gbit} / \mathrm{s}$ for NRZ measurements for all the lasers shown in Fig. 4. By tuning the photon lifetime the energy consumption at $50 \mathrm{Gbit} / \mathrm{s}$ has been decreased by $25 \%$ [3]. We assume, that all these values do not present the true limits of our devices.

Presently for the new IEEE 802.3 standards lasers emitting at two or all four of the wavelengths of $850 \mathrm{~nm}, 880 \mathrm{~nm}, 910$ $\mathrm{nm}$, and $940 \mathrm{~nm}$, are under discussion enabling a quadrupling of the data rate across the same optical fiber by using lasers with similar properties. [15] Showing a bandwidth close to $30 \mathrm{GHz}$ and side-mode suppression ratios of $35 \mathrm{~dB}$ from close to threshold till thermal rollover our lasers have the potential for further increasing the transmission distance beyond 100 $\mathrm{m}$, and/or the bitrates beyond $50 \mathrm{Gbit} / \mathrm{s}$.

\subsection{Data transmission experiments}

The purpose of data transmission experiments is to demonstrate the performance of a device under test in a data link like environment.

The signal, here a pseudorandom binary sequence with a word length of 27-1, was generated by a SHF $12100 \mathrm{~B}$ bit pattern generator (BPG). The electrical signal provided by that bit pattern generator was evaluated by a SHF $11100 \mathrm{~B}$ error analyzer, showing that an error free signal can be generated up to $52 \mathrm{Gbit} / \mathrm{s}$.

The BPG was followed by a $+8 \mathrm{~dB}$ amplifier (SHF $801 \mathrm{P}$ ) driving the VCSEL through a bias-tee. The VCSELs are mounted on a temperature-controlled probe station. The VCSEL emission is butt-coupled to the cleaved end of a $2 \mathrm{~m}-$ long novel OM5 multimode fiber (MMF), to ensure $M(f, P)$ is independent of the emission wavelength.

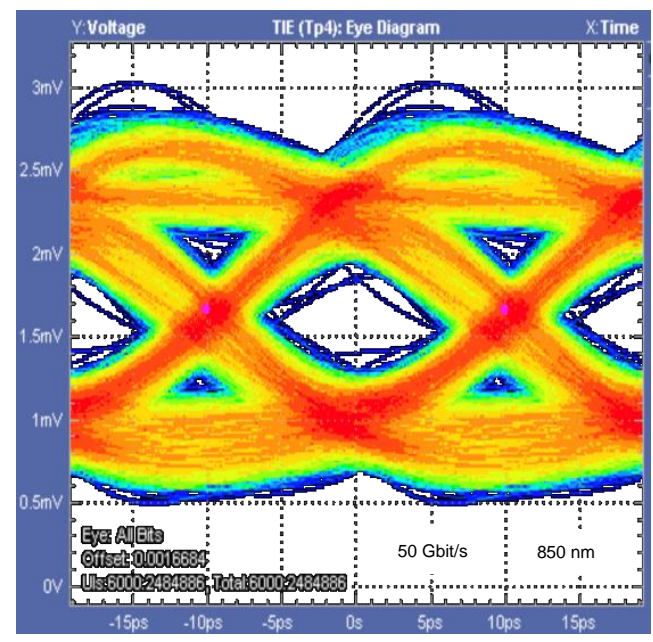

Fig. 3: The eye diagram (left) for a back-to-back data rate of $50 \mathrm{Gbit} / \mathrm{s}$ for an $\sim 3 \mu \mathrm{m}$ oxide aperture diameter VCSEL emitting at $850 \mathrm{~nm}$, which is representative of all our devices. 
To characterize the single mode VCSELs at $850 \mathrm{~nm}, 880 \mathrm{~nm}, 910 \mathrm{~nm}$ and $940 \mathrm{~nm}$ without changing the receiver we used a $33 \mathrm{GHz}$ optical probe (Tektronix DPO7OE1) with large sensitivity and low noise in a wide band from $750 \mathrm{~nm}$ to 1650 $\mathrm{nm}$. The optical probe perfectly matches the Tektronix $70 \mathrm{GHz}$ real time oscilloscope (DPS77004SX). To compensate the disadvantage of the linear amplifier in the optical probe by receiving non-return to zero (NRZ) bit patterns we have used software filtering provided by the oscilloscope.

Fig. 3 shows the result of a non-return to zero data transmission experiment across the $2 \mathrm{~m}$ OM5 MMF which has been used at all wavelengths. Fig. 3 has been taken with the Tektronix $70 \mathrm{GHz}$ real time oscilloscope and the $33 \mathrm{GHz}$ optical probe. Since the lasers at all wavelengths show very similar bandwidths and output power, the data transmission results are the same. The eye diagram and BER of an $850 \mathrm{~nm}$ laser shown here is representative for all such lasers. By multiplexing the wavelengths, a transmission rate exceeding $200 \mathrm{Gbit} / \mathrm{s}$ can be achieved on a single multimode fiber.

\section{CONCLUSION}

VCSELs for 200+ Gbit/s data transmission via multiplexing directly modulated newly designed VCSELs emitting at 850 $\mathrm{nm}, 880 \mathrm{~nm}, 910 \mathrm{~nm}, 940 \mathrm{~nm}$ are presented. The large linearity of the L-I-characteristics of our devices will easily allow still larger data rates via, for example PAM 4, up to $400 \mathrm{Gbit} / \mathrm{s}$. The energy efficiency expressed by the HBR is as low as $240 \mathrm{fJ} / \mathrm{bit} @ 50 \mathrm{~Gb} / \mathrm{s}$. Due to the large optical power, already for single mode emission, our VCSELs are well suited for long distance OM5 optical fiber transmission, at least up to $100 \mathrm{~m}$, in the future most probably beyond this distance. Thus strategic input for the ongoing discussions of the IEEE $802.3 \mathrm{~cd}$ and $802.3 \mathrm{~cm}$ task forces on standards for next generation physical layers of $400 \mathrm{~Gb} / \mathrm{s}$ Datacom networks is presented here.

\section{ACKNOWLEDGEMENTS}

GL is grateful for the hospitality of TUB and DFG via SFB 787 during part of this work. He appreciates the support of CAS via the Hundred Talents Program. The authors appreciate the support of James A. Lott. R. Rosales was helping to acquire the data of Fig.3. Corning supplied the fiber and Tektronix the $70 \mathrm{GHz}$ real time oscilloscope used here.

\section{REFERENCES}

[1] G. Larisch.et al. IEEE J Sel. Topics QE submitted

[2] P. Wolf, P. Moser, G. Larisch, W. Hofmann, and D. H. Bimberg, "High-Speed and Temperature-Stable oxide-confined 980-nm VCSELs for Optical Interconnects," IEEE Journal of Selected Topics in Quantum Electronics, vol. 19, no. 4, pp. 1-7, 2013.

[3] G. Larisch, P. Moser, J. A. Lott, and D. Bimberg, "Large Bandwidth, Small Current Density, and Temperature Stable 980-nm VCSELs," IEEE Journal of Quantum Electronics, vol. 53, no. 6, pp. 1-8, 2017.and

[4] D.Bimberg, G. Larisch, J.A.Lott US Patent 9,979,158 B1

[5] J. M. Dallesasse, N. Holonyak, A. R. Sugg, T. A. Richard, and N. El-Zein, "Hydrolyzation oxidation of \AlGaAsGaAs quantum well heterostructures and superlattices," Applied Physics Letters, vol. 57, no. 26, p. 2844, 1990.

[6] D. Kuchta et al., "A 56.1Gb/s NRZ Modulated 850nm VCSEL-Based Optical Link," in Optical Fiber Communication Conference, p. OW1B.5.

[7] C. L. Schow, A. V. Rylyakov, C. W. Baks, F. E. Doany, and J. A. Kash, "25-Gb/s 6.5-pJ/bit 90-nm CMOS-Driven Multimode Optical Link," IEEE Photonics Technology Letters, vol. 24, no. 10, pp. 824-826, 2012.

[8] J. E. Proesel, B. G. Lee, C. W. Baks, and C. L. Schow, "35-Gb/s VCSEL-Based optical link using 32-nm SOI CMOS circuits," in Optical Fiber Communication Conference (OFC/NFOEC), $2013\{$ Los Angeles, CA, USA\}, 2013, pp. 13.

[9] D. Schoeniger, R. Henker, and F. Ellinger, "An 850-nm common-cathode VCSEL driver with tunable energy efficiency for $45 \mathrm{Gbit} / \mathrm{s}$ data transmission without equalization," in 2017 IEEE Asia Pacific Microwave Conference (APMC), 2017, pp. 1103-1106.

[10] V. Shchukin et al., "Single-Mode Vertical Cavity Surface Emitting Laser via Oxide-Aperture-Engineering of Leakage of High-Order Transverse Modes," IEEE Journal of Quantum Electronics, vol. 50, no. 12, pp. 990-995, 2014. 
[11] P. Moser et al., "81 fJ/bit energy-to-data ratio of $850 \mathrm{~nm}$ vertical-cavity surface-emitting lasers for optical interconnects," Applied Physics Letters, vol. 98, no. 23, pp. 231106-1-3, 2011.

[12] L. A. Coldren and S. W. Corzine, Diode lasers and photonic integrated circuits (Wiley series in microwave \& optical engineering). New York, NY, USA: Wiley, 1995, pp. xxiii, 594.

[13] C. E. Shannon, "Communication in the Presence of Noise," Proceedings of the IRE, vol. 37, no. 1, pp. 10-21, 1949.

[14] H. Li, P. Wolf, P. Moser, G. Larisch, J. A. Lott, and D. H. Bimberg, "Temperature-Stable, Energy-Efficient, and HighBit Rate Oxide-Confined $980 \mathrm{~nm}$ VCSELs for Optical Interconnects," IEEE Journal of Selected Topics in Quantum Electronics, vol. 21, no. 6, p. 1700409, 2015.

[15]P. Moser, J. A. Lott, G. Larisch, and D. H. Bimberg, "Impact of the Oxide-Aperture Diameter on the EnergyEfficiency, Bandwidth, and Temperature Stability of $980 \mathrm{~nm}$ VCSELs," Journal of Lightwave Technology, vol. 33, no. 4, pp. 825-831, 2015.

[16] N. N. Ledentsov et al., "Progress on single mode VCSELs for data- and tele-communications," in Proc. of SPIE 8276, Vertical-Cavity Surface-Emitting Lasers XVI, 2012. 